\title{
氟噻唑吡乙酮类衍生物的合成及杀菌活性
}

\author{
丁成荣 ${ }^{a, b}$ 潘亚运 $a, b$ 殷 许 ${ }^{a}$ 谭成侠 $*, a$ 王学东*, $b$ \\ ( ${ }^{a}$ 浙江工业大学化学工程学院 杭州 310032) \\ ( ${ }^{b}$ 温州医科大学公共卫生与管理学院 浙江省流域水环境与健康风险研究重点实验室＼cjkstart温州 325035)
}

\begin{abstract}
摘要 为探究具有生物活性的先导化合物，以氟噻唑吡乙酮为模板，设计并合成了 16 个未见文献报道的氟噻唑吡乙酮 类衍生物, 初步研究了在噻唑环上与硫相邻碳(5 号碳)上取代基的变化对抑菌活性的影响. 其结构经 ${ }^{1} \mathrm{H} N \mathrm{NMR},{ }^{13} \mathrm{C} N \mathrm{NR}$ 和 HRMS 确证, 测试表明: 在 $100 \mu \mathrm{g} / \mathrm{mL}$ 的浓度下目标化合物普遍具有杀菌活性, 其中 1-(4-(4-环丙基-5-(2-氟苯基)噻 唑-2-基)哌啶-1-基)-2-(5-甲基-3-三氟甲基- $1 H$-吡唑-1-基)乙-1-酮(9o)对小麦赤霉病的抑制率为 $60 \%$; 6 个化合物对苹果褐 斑病的抑制率为 70\%; 4 个化合物对对马铃薯晚疫病的抑制率为 50\%; 2-(5-甲基-3-三氟甲基-1 $H$-吡唑-1-基)-1-(4-(4-甲 基-5-(间甲基苯基)噻唑-2-基)哌啶-1-基)乙-1-酮(9h)对黄瓜灰霰病的抑制率为 75\%. 并且在 $100 \mu \mathrm{g} / \mathrm{mL}$ 浓度下目标化合 物对苹果褐斑病、黄瓜灰霉病抑制效果. 要高于对照药品嘧菌酯在 $50 \mu \mathrm{g} / \mathrm{mL}$ 时的抑菌活性.
\end{abstract}

关键词＼cjkstart氟噻唑吡乙酮; 噻唑; 杀菌活性

\section{Synthesis and Fungicidal Activity of Novel Oxathiapiprolin Derivatives}

\author{
Ding, Chengrong ${ }^{a, b} \quad$ Pan, Yayun ${ }^{a, b} \quad$ Yin, $\mathrm{Xu}^{a} \quad$ Tan, Chengxia ${ }^{*, a} \quad$ Wang, Xuedong ${ }^{*, b}$ \\ ( ${ }^{a}$ College of Chemical Engineering Science, Zhejiang University of Technology, Hangzhou 310032) \\ ( ${ }^{b}$ Zhejiang Provincial Key Laboratory of Watershed Science and Health, College of Public Health and Management, \\ Wenzhou Medical University, Wenzhou 325035)
}

\begin{abstract}
In order to explore the structure of lead compounds with biological activities, using oxathiapiprolin as a template, sixteen oxathiapiprolin derivatives were designed and synthesized to study the influences of substituent to the fungicidal activities which connected with carbon (No. 5 carbon) near the sulfur on the thiazole ring. All the structures were confirmed by ${ }^{1} \mathrm{H}$ NMR, ${ }^{13} \mathrm{C}$ NMR and HRMS. Preliminary bioassay showed that the target compounds generally had fungicidal activitives in vitro at a concentration of $100 \mu \mathrm{g} / \mathrm{mL}$, the fungicidal activities of 1-(4-(4-cyclopropyl-5-(2-fluorophenyl)thiazol-2-yl)piperidin1-yl)-2-(5-methyl-3-trifluoromethyl-1H-pyrazol-1-yl)ethan-1-one (9o) against Fusarium graminearum was $60 \%$, the fungicidal activities of six target compounds against Diplocarpon mali were $70 \%$, the fungicidal activities of four target compounds against Phytophthora infestans were 50\%, and the fungicidal activities of 2-(5-methyl-3-trifluoromethyl-1H-pyrazol-1yl)-1-(4-(4-methyl-5-(m-tolyl)thiazol-2-yl)piperidin-1-yl)ethan-1-one (9h) against Botrytis cinerea was 75\%. In addtion, the fungicidal activities of the target compounds aganist Diplocarpon mali and Botrytis cinerea at $100 \mu \mathrm{g} / \mathrm{mL}$ were higher to azoxystrobin at $50 \mu \mathrm{g} / \mathrm{mL}$.
\end{abstract}

Keywords oxathiapiprolin; thiazole; fungicidal activity

\section{Introduction}

Oxathiapiprolin (trade name ZorvecR) was the first piperidinyl thiazole isoxazoline fungicide developed by DuPont. ${ }^{[1]}$ It was low in toxicity and high in efficiency (the dosage was $1 / 5 \sim 1 / 100$ of common fungicides), especially for the pathogens of Oomycetes. ${ }^{[2 \sim 6]}$ Besides oxysterol-binding protein (OSBP) was target protein. ${ }^{[7]}$ Therefore oxathiapiprolin didn't have mutual resistance with other fungicides. Since ZorvecR was sold, sales had increased year by year. DuPont, Bayer, Syngenta and other researchers in the field had derived and reported many structures of oxathiapiprolin derivatives ${ }^{[8 \sim 16]}$ owning fungicidal activities. For example, the fungicidal activity of derivative $\mathbf{1}^{[17]}$ developed by DuPont against Plasmopara viticola and Phytophthora infestans was $100 \%$ at $40 \mu \mathrm{g} / \mathrm{mL}$, that of

* Corresponding authors. E-mail: tanchengxia@zjut.edu.cn; 1521554541@qq.com

Received January 7, 2019; revised February 27, 2019; published online March 29, 2019.

Project supported by the Collaborative Innovation Center of Zhejiang Province Green Pesticide

浙江省绿色农药 2011 协同创新中心开放基金资助项目. 
derivative $2^{[18]}$ developed by Bayer against Pseudoperonos pora cubensis Rostov was $100 \%$ at $100 \mu \mathrm{g} / \mathrm{mL}$, that of derivative $3^{[19]}$ developed by the Clemens Lamberth group against Phytophthora infestans and Plasmopara viticola was $100 \%$ at $6 \mu \mathrm{g} / \mathrm{mL}$ (Figure 1). The derivative $4^{[20,21]}$ developed by the Won-Sik Choi group had both fungicidal and insecticidal activity (Mosquito larvae) with $\mathrm{LC}_{50}$ (Lethal concentration 50$)=0.513 \mathrm{mmol} \cdot \mathrm{L}^{-1}$. Excitingly, another piperidinylthiazole isoxazoline fungicide was awarded the ISO (International Organization for Standardization) common name fluoxapiprolin in October 2018. The compound was similar with oxathiapiprolin containing five ring structures (benzene, isoxazole, thiazole, piperidine and pyrazole) and highly fungicidal activity. For example, the fungicidal activity of fluoxapiprolin against Phytophthora infestans and Plasmopara viticola was more than $75 \%$ at $0.2 \mu \mathrm{g} / \mathrm{mL}^{[22,23]}$

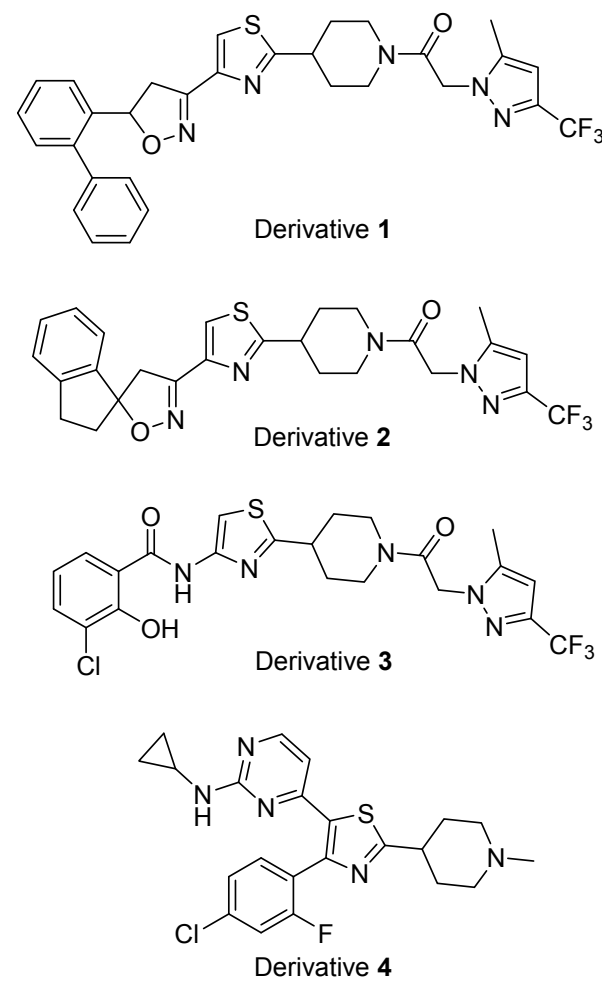

Figure 1 Structures of derivatives $1 \sim 4$

Thiazoles ${ }^{[24,25]}$ had always been a hot spot in the development of new pesticides. A series of compounds were produced with a broad spectrum of biological activity by modifying structures when the thiazole groups were introduced into a variety of different compound structures. Thiazolidine piperidine structure was a novel thiazolecontaining structure which had received much attention in recent years, and substituent can be introduced at positions 4 and 5 on the thiazole ring. However the research about 5 -substituted compounds was dwarfed compared with 4-substituted compounds. In order to study the influences of substituent to fungicidal activities about 5-substituted compounds, our group designed and synthesized a series of novel oxathiapiprolin derivatives showed in Figure 2. The structures were confirmed by ${ }^{1} \mathrm{H}$ NMR, ${ }^{13} \mathrm{C}$ NMR and HRMS. Then, the preliminary bioactivity tests showed that target compounds had fungicidal activities (Table 1). The synthetic route of the target compounds was shown in the Scheme 1.
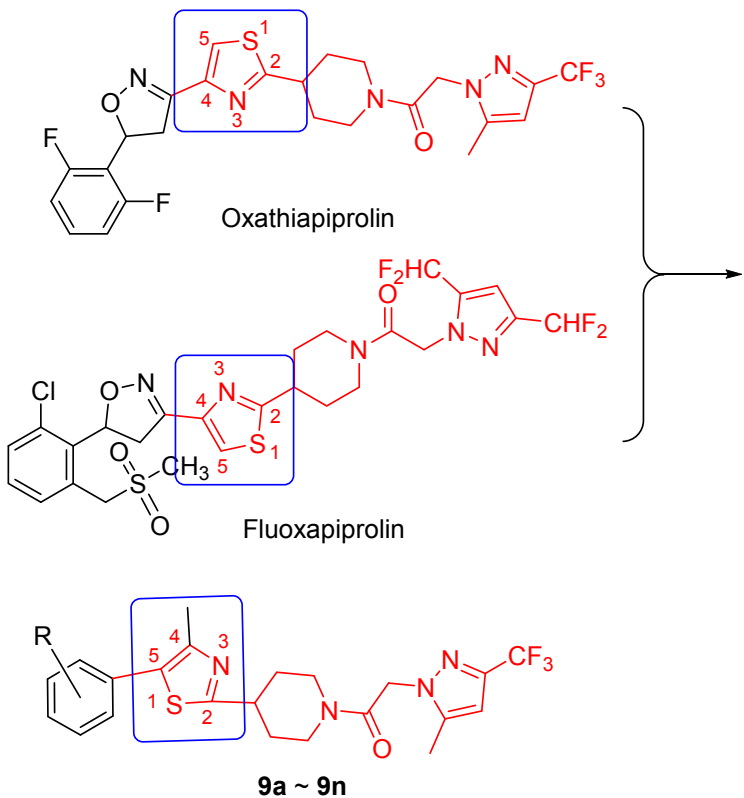

Figure 2 Design of target compounds

\section{Results and discussion}

\subsection{Synthesis and characterization}

Substituted aniline as a substrate was experienced diazotization, ${ }^{[26]}$ chlorination, ${ }^{[27]}$ cyclization and deprotection ${ }^{[28]}$ to intermediate $\mathbf{8}$, and then reacted with intermediate $\mathbf{3}^{[29,30]}$ to give the target compounds $9 \mathbf{9} \sim 9 \mathbf{p}$. However, the synthesis of intermediate $\mathbf{4}$ and intermediate $\mathbf{5}$ largely inhibited the diversity of substrates. The synthesis of intermediate 4 was influenced by the mechanism of diazotization, and the synthesis of intermediate $\mathbf{5}$ had multiple substitutions and competitive reaction. For that, the multiple substitutions could be controlled by adjusting the amount of sulfonyl chloride, but the competitive reaction was failed to control. It was not conducive to form intermediate $\mathbf{5}$ when ortho substituent or strong electron donating group existed on benzene ring. Besides, the by-product was dominant in the case of ortho-disubstituted structure. Therefore the target compound 9p (No. 4 carbon) was different with 9k, 91 and $9 \mathrm{~m}$ (No. 5 carbon) on the position of the thiazole ring showed in Scheme 1.

Spectral analysis was carried out using the target compound 9a as an example. In ${ }^{1} \mathrm{H}$ NMR spectrum, 23 hydrogens were assigned. $\delta 7.47 \sim 7.40(4 \mathrm{H})$ and $7.38 \sim 7.33$ $(1 \mathrm{H})$ were the proton signals of phenyl, $\delta 6.35(1 \mathrm{H})$ was the proton signal on the pyrazole ring, $\delta 5.01(2 \mathrm{H})$ was the proton signal of methylene linked with pyrazole, $\delta 2.48$ $(3 \mathrm{H})$ was the proton signal of the methyl group on the thiazole ring, $\delta 2.33(3 \mathrm{H})$ was the proton signal of the methyl 
<smiles>CCOC(=O)CCl</smiles>

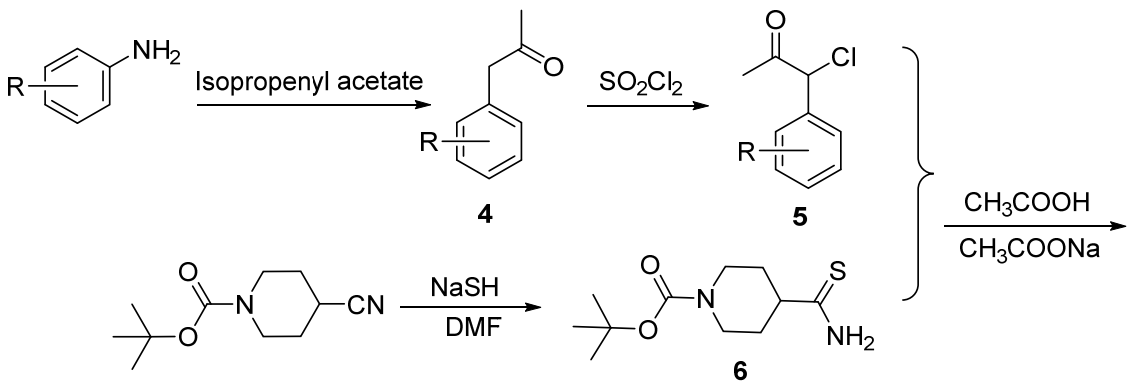
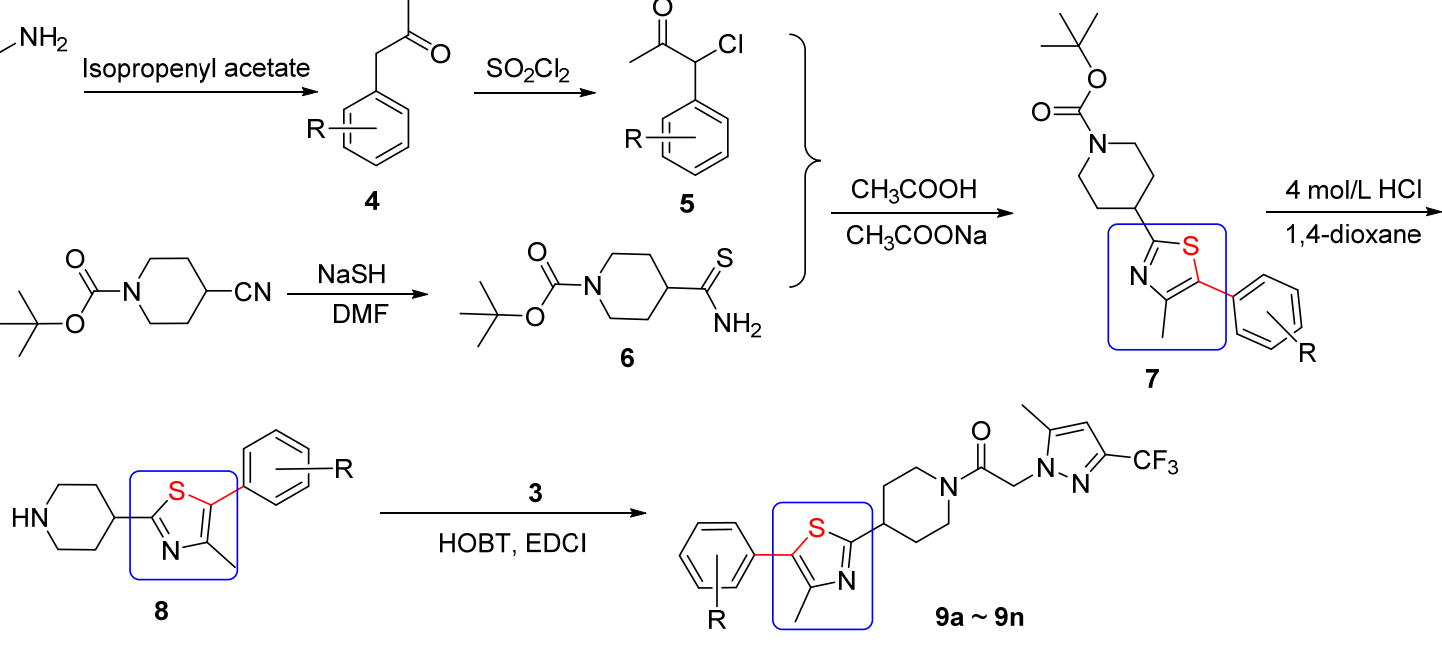

9a: $\mathrm{R}=\mathrm{H} ; 9 \mathrm{~b}: \mathrm{R}=4-\mathrm{CF}_{3} ;$ 9c: $\mathrm{R}=4-\mathrm{F} ; 9 \mathrm{~d}: \mathrm{R}=4-\mathrm{Cl} ; 9 \mathrm{e}: \mathrm{R}=4-\mathrm{Br} ;$ 9f: $\mathrm{R}=4-\mathrm{Bu}-t ; 9 \mathrm{~g}: \mathrm{R}=3-\mathrm{Cl} ; 9 \mathrm{~h}: \mathrm{R}=3-\mathrm{CH}_{3}$ 9i: $R=3-\mathrm{CF}_{3} ; 9 j: \mathrm{R}=3-\mathrm{Br} ; 9 \mathrm{k}: \mathrm{R}=2,3-\mathrm{Cl}_{2} ; 9 \mathrm{I}: \mathrm{R}=2,4-\mathrm{Cl}_{2} ; 9 \mathrm{~m}: \mathrm{R}=2,5-\mathrm{Cl}_{2} ; 9 \mathrm{n}: \mathrm{R}=2-\mathrm{F}$;<smiles>Cc1cc(C(F)(F)F)nn1CC(=O)N1CCC(c2nc(C3CC3)c(-c3ccccc3F)s2)CC1</smiles><smiles>Cc1cc(C(F)(F)F)nn1CC(=O)N1CCC(c2nc(Cc3c(Cl)cccc3Cl)cs2)CC1</smiles>

Scheme 1 Synthetic route of target compounds

group on the pyrazole ring, and the remaining $\delta 4.62(1 \mathrm{H})$, $4.06(1 \mathrm{H}), 3.37 \sim 3.20(2 \mathrm{H}), 2.98 \sim 2.83(1 \mathrm{H}), 2.22(2 \mathrm{H})$ and $1.87 \sim 1.72(2 \mathrm{H})$ were the proton signals of the piperidine ring. In the ${ }^{13} \mathrm{C}$ NMR spectrum, 22 carbons were assigned. There were overlapping carbons due to the symmetry relationship of benzene rings: $\delta 129.12(2 \times \mathrm{C})$ and $128.64(2 \times \mathrm{C})$, and the pyrazole ring was affected by trifluoromethyl group: $\delta 121.29$ (q, $J=268.4 \mathrm{~Hz}), 141.74$ (q, $J=37.8 \mathrm{~Hz}$ ) and 104.31 104.04 (m). The molecular ion peak of this compound was calculated as $[\mathrm{M}+\mathrm{H}]^{+}$ 449.1617, found 449.1615, and the absolute error was within 0.003 .

\subsection{Fungicidal activity}

The fungicidal activities of the target compounds $9 \mathbf{a} \sim 9 \mathbf{p}$ were evaluated against Fusarium graminearum ( $F$. graminearum), Diplocarpon mali (D. mali), Rhizoctonia solani Ktihn (R. solani Ktihn), Phytophthora infestans (P. infestans), Botrytis cinerea (B. cinerea) at $100 \mu \mathrm{g} / \mathrm{mL}$. The results were listed in Table 1 . It was found that all the target compounds exhibited certain fungicidal activity against the tested fungi at $100 \mu \mathrm{g} / \mathrm{mL}$. On the whole, the target compounds showed higher fungicidal activities to azoxystrobin at $50 \mu \mathrm{g} / \mathrm{mL}$ against Diplocarpon mali and Botrytis cinerea. Besides the fungicidal activities of $\mathbf{9 b}, \mathbf{9 c}$, 9e and $9 \mathrm{~h}$ were more than $70 \%$. In addition, it showed that the disubstituted structures $(\mathbf{9 k}, 9 \mathbf{9}, 9 \mathrm{~m})$ could not effectively increase the fungicidal activities comparing with the others $(\mathbf{9 a} \sim 9 \mathbf{j})$. Then there was no significant difference in fungicidal activity between para-substituted structures $(\mathbf{9 b} \sim 9 \mathbf{9})$ and meta-substituted structures $(\mathbf{9 g} \sim 9 \mathbf{j})$. Furthermore, the structure of $\mathbf{9 h}\left(3-\mathrm{CH}_{3}\right)$ was the best when compared with others $(\mathbf{9 a} \sim 9 \mathrm{~g}, 9 \mathbf{9} \sim 9 \mathbf{p})$. On the other hand, Major target compounds against Fusarium graminearum were $20 \sim 40 \%$, and the fungicidal activities of $9 \mathbf{b}$, 9g, 9h and 9i were 40\%. However, 9o (60\%) was obviously higher to other target compounds (below 40\%) against Fusarium graminearum and the structure of 90 was similar to $9 \mathbf{n}$. Therefore the appearance of cyclopropyl on the thiazole ring might improve fungicidal activity against Fusarium graminearum. In addition the target compounds against Phytophthora infestans were $20 \sim 50 \%$, and the fungicidal activities of $9 a, 9 b, 9 c$ and $9 e$ were $50 \%$. Besides Phytophthora infestans was influenced more by the kinds of substituents when compared with other fungi. Moreover the results of substituted benzene was better to benzene (9a) against in Botrytis cinerea when compared with $9 b \sim 9 n$. 
Table 1 Fungicidal activity (inhibition rate/\%) of the target compounds $\mathbf{9 a} \sim \mathbf{9 p}$ at $100 \mu \mathrm{g} / \mathrm{mL}$

\begin{tabular}{|c|c|c|c|c|c|c|}
\hline Compd. & $\mathrm{R}$ & F. graminearum & D. mali & R. solani Ktihn & P. infestans & B. cinerea \\
\hline $9 a$ & $\mathrm{H}$ & 30 & 70 & 0 & 50 & 30 \\
\hline $9 \mathrm{~b}$ & $4-\mathrm{CF}_{3}$ & 40 & 70 & 0 & 50 & 70 \\
\hline $9 c$ & $4-F$ & 20 & 70 & 0 & 50 & 70 \\
\hline 9d & $4-\mathrm{Cl}$ & 30 & 0 & 0 & 0 & 70 \\
\hline $9 e$ & $4-\mathrm{Br}$ & 20 & 70 & 0 & 50 & 70 \\
\hline 9f & $4-{ }^{t} \mathrm{Bu}$ & 30 & 0 & 0 & 0 & 60 \\
\hline $9 g$ & $3-\mathrm{Cl}$ & 40 & 50 & 0 & 0 & 65 \\
\hline $9 \mathrm{~h}$ & $3-\mathrm{CH}_{3}$ & 40 & 70 & 0 & 50 & 75 \\
\hline $9 \mathrm{i}$ & $3-\mathrm{CF}_{3}$ & 40 & 70 & 0 & 40 & 60 \\
\hline $9 j$ & $3-\mathrm{Br}$ & 0 & 0 & 0 & 0 & 10 \\
\hline $9 k$ & $2,3-\mathrm{Cl}_{2}$ & 10 & 60 & 0 & 20 & 40 \\
\hline 91 & $2,4-\mathrm{Cl}_{2}$ & 30 & 60 & 0 & 30 & 60 \\
\hline $9 m$ & $2,5-\mathrm{Cl}_{2}$ & 20 & 60 & 0 & 30 & 60 \\
\hline $9 n$ & $2-\mathrm{F}$ & 0 & 0 & 0 & 0 & 20 \\
\hline 90 & - & 60 & 50 & 0 & 0 & 50 \\
\hline $9 p$ & - & 20 & 55 & 0 & 20 & 40 \\
\hline Azoxystrobin & - & 80 & 50 & 60 & 100 & 40 \\
\hline
\end{tabular}

${ }^{a}$ Azoxystrobin as a control drug at a concentration of $50 \mu \mathrm{g} / \mathrm{mL}$.

\section{Conclusions}

Using oxathiapiprolin as a template, sixteen oxathiapiprolin derivatives were designed and synthesized to study the influences of substituent about the fungicidal activities which connected with carbon (No. 5 carbon) near the sulfur on thiazole ring. The structures were confirmed by ${ }^{1} \mathrm{H}$ NMR, ${ }^{13} \mathrm{C}$ NMR and HRMS. From the preliminary bioactivity tests at $100 \mu \mathrm{g} / \mathrm{mL}$, the target compounds showed universal fungicidal activities and showed higher fungicidal activity against Diplocarpon mal and Botrytis cinereal compared with the azoxystrobin at $50 \mu \mathrm{g} / \mathrm{mL}$. However, while compared it with the commercial oxathiapiprolin, a significant difference in efficacy was observed. Later, the pyridine structures were prepared to replace the benzene structures in order to study the difference about the position of $\mathrm{N}$ on pyridine ring.

\section{Experimental section}

\subsection{Instruments}

Melting points were defined by an X-4 apparatus and uncorrected. ${ }^{1} \mathrm{H}$ NMR spectra were measured on a Bruker AV-600 instrument using $\mathrm{CDCl}_{3}$ as the solvent. Mass spectra were determined on a a Bruker Daltonics Bio-TOF-Q III mass spectrometer (ESIMS). 1-Cyclopropyl-2-(2-fluorophenyl)ethan-1-one (4o) and other reagents purchased were analytical grade or recently prepared before use. The processes of reactions were grasped by TLC, and the silica gel of TLC was GF254.

\subsection{Synthesis}

4.2.1 Preparation of 3-methyl-5-trifluoromethyl-1 $H$ pyrazole (1)

A mixture of methyl 1,1,1-trifluoropentane-2,4-dione $(0.1 \mathrm{~mol})$, hydrazine hydrate $(0.1 \mathrm{~mol})$ and EtOH $(30 \mathrm{~mL})$ was stirred at reflux for $6 \mathrm{~h}$. The reaction was concentrated in vacuum to give 5-methyl-3-trifluoromethyl-1H-pyrazole (1) directly used in the next reaction, yield 97.6\%, light yellow solid.

4.2.2 Preparation of ethyl 2-(3-methyl-5-(trifluoromethyl)-1H-pyrazol-1-yl)acetate (2)

A mixture of 3-methyl-5-trifluoromethylpyrazole (1) (0.1 mol), ethylchloroacetate $(0.2 \mathrm{~mol})$, potassium carbonate $(0.3 \mathrm{~mol})$ and $N, N$-dimethylformamide $(80 \mathrm{~mL})$ was stirred at ambient temperature overnight. The orange mixture was filtered, diluted with ethylacetate, washed with water and brine, dried over with $\mathrm{MgSO}_{4}$ and concentrated under reduced pressure to give 2-(5-methyl-3-(trifluoromethyl)-1H-pyrazol-1-yl)acetate (2) directly used in the next reaction, yield $80.6 \%$, light yellow liquid.

4.2.3 Preparation of 5-methyl-3-trifluoromethyl-1Hpyrazol-1-acetic acid (3)

2-(5-Methyl-3-trifluoromethyl-1H-pyrazol-1-yl)acetate (2) $(0.1 \mathrm{~mol})$, THF $(100 \mathrm{~mL})$ and $50 \%$ aqueous $\mathrm{NaOH}$ solution $(30 \mathrm{~mL})$ were mixed and stirred at ambient temperature overnight. When the reaction was over, the THF was removed under reduced pressure and the aqueous solution was washed with ethylacetate. Then the aqueous layer was acidified with $10 \% \mathrm{HCl}$ solution to $\mathrm{pH}=1$ to give a precipitate. The precipitate was filtered, washed with water and dried to give 5-methyl-3-trifluoromethyl-1H-pyrazol1 -acetic acid (3) directly used in the next reaction, yield $85.2 \%$, a white solid.

4.2.4 Preparation of 1-(substituted-phenyl)propan-2ones $(\mathbf{4 a} \sim \mathbf{4 n}$ and $\mathbf{4 p})$

Synthesis by reference: ${ }^{[25]}$ A mixture of substituted aniline $(0.15 \mathrm{~mol})$, aqueous $\mathrm{HCl}$ solution $(38 \mathrm{~mL})$ and water $(55 \mathrm{~mL})$ was cooled to $-5{ }^{\circ} \mathrm{C}$ in an ice-salt bath, and a cold solution of sodium nitrite $(0.15 \mathrm{~mol})$ in water $(15 \mathrm{~mL})$ was added over $45 \mathrm{~min}$ with stirring. The temperature of the reaction mixture was held at $-5 \sim 2{ }^{\circ} \mathrm{C}$. The mixture 
was stirred for an additional $15 \mathrm{~min}$, and a cold solution boric acid $(0.19 \mathrm{~mol})$ in $40 \%$ of hydrofluoric acid $(39 \mathrm{~mL})$ was slowly added with stirring. The mixture was stirred for an additional $30 \mathrm{~min}$ and then stored at about $0{ }^{\circ} \mathrm{C}$ for $2 \mathrm{~h}$. The mixture was filtered, and the solid was washed with $95 \%$ ethanol $(30 \mathrm{~mL})$ to give the colorless salt.

To a stirred mixture of anhydrous $\mathrm{CH}_{3} \mathrm{COONa}(0.25$ $\mathrm{mol})$, cuprous oxide $(0.013 \mathrm{~mol})$, and isopropenyl acetate $(52.6 \mathrm{~mL})$, the colorless salt was added over $30 \mathrm{~min}$, while the temperature of the reaction mixture was held at $20 \sim$ $25{ }^{\circ} \mathrm{C}$. The reaction mixture was stirred an additional $6 \mathrm{~h}$ at $20 \sim 25{ }^{\circ} \mathrm{C}$. The mixture was filtered and washed with ethylacetate. The solution was collected and distilled under vacuum to give 1-(substituted-phenyl)propan-2-ones (4a $4 \mathbf{n}, 4 \mathbf{p})^{[25]}$ as a clear yellow liquid, yields $25 \% \sim 60 \%$, influenced by the mechanism of diazotization.

4.2.5 Preparation of 1-chloro-1-(substituted-phenyl)propan-2-ones $(\mathbf{5 a} \sim \mathbf{5 p})$

In a $50 \mathrm{~mL}$ round bottom flask, 1-(substituted-phenyl)propan-2-ones $(\mathbf{4 a} \sim \mathbf{4 p})(0.02 \mathrm{~mol})$ were dissolved in dichloromethane $(15 \mathrm{~mL})$. This solution was cooled to $0{ }^{\circ} \mathrm{C}$, and sulfuryl chloride $(0.022 \mathrm{~mol})$ was added slowly over $1 \mathrm{~h}$. The reacting solution was stirred for $7 \mathrm{~h}$ at room temperature until TLC revealed complete reaction. Water $(20 \mathrm{~mL})$ was added to the solution, the aqueous layer was separated and extracted with dichloromethane $(2 \times 10 \mathrm{~mL})$. Then the combined organic layers were washed with saturated $\mathrm{NaCl}$ solution, dried over anhydrous $\mathrm{MgSO}_{4}$ and evaporated to give the crude 1-chloro-1-(substituted-phenyl)propan-2-ones $(\mathbf{5 a} \sim \mathbf{5 p})$ directly used in the next reaction.

4.2.6 Preparation of tert-butyl 4-carbamothioylpiperidine-1-carboxylate $(6)$

In a $250 \mathrm{~mL}$ round bottom flask, 1-Boc-4-cyanopiperidine $(0.04 \mathrm{~mol}), 70 \% \mathrm{NaSH}(0.18 \mathrm{~mol})$ and $\mathrm{NH}_{4} \mathrm{Cl}(0.18$ $\mathrm{mol})$ were dissolved in DMF $(120 \mathrm{~mL})$. The mixture reacted at room temperature with string for $72 \mathrm{~h}$. Ice water $600 \mathrm{~g}$ ) was added in $1 \mathrm{~L}$ beaker, and then the reacted solution was added slowly in beaker with stirring to give a precipitate. The precipitate was filtered, washed with water and dried to give tert-butyl 4-carbamothioylpiperidine-1carboxylate (6), yield 95.2\%, a white solid. m.p. 133 $134{ }^{\circ} \mathrm{C}$; ${ }^{1} \mathrm{H}$ NMR (500 MHz, Chloroform-d) $\delta: 7.66$ (s, $1 \mathrm{H}), 7.10(\mathrm{~s}, 1 \mathrm{H}), 4.22(\mathrm{~d}, J=12.5 \mathrm{~Hz}, 2 \mathrm{H}), 2.78 \sim 2.65$ (m, 3H), 1.90 (d, $J=12.6 \mathrm{~Hz}, 2 \mathrm{H}), 1.73$ (qd, $J=12.9,4.5$ $\mathrm{Hz}, 2 \mathrm{H}), 1.46$ (s, 9H).

4.2.7 Preparation of tert-butyl 4-(4-methyl-5-(substituted-phenyl)thiazol-2-yl)piperidine-1-carboxylates $(7 \mathbf{a} \sim 7 \mathbf{p})$

In a $250 \mathrm{~mL}$ round bottom flask, tert-butyl 4-carbamothioylpiperidine-1-carboxylate (6) $(0.05 \mathrm{~mol})$, anhydrous $\mathrm{CH}_{3} \mathrm{COONa}(0.25 \mathrm{~mol})$ and 1-(substituted-phenyl)propan2-ones $(\mathbf{4 a} \sim \mathbf{4 p})(0.05 \mathrm{~mol})$ were dissolved in glacial acetic acid $(100 \mathrm{~mL})$. The mixture reacted at $85{ }^{\circ} \mathrm{C}$ with stirring for $12 \mathrm{~h}$ until TLC revealed complete reaction. The glacial acetic acid was removed under reduced pressure, and the remaining liquid was extracted with ethylacetate (100 $\mathrm{mL} \times 3)$. The organic layer was held, washed with saturated $\mathrm{NaHCO}_{3}$ solution, brine and dried over $\mathrm{MgSO}_{4}$. Finally the organic solution was concentrated under reduced pressure and separated by column to give 7 directly used in the next reaction.

$7 \mathbf{g}$ as an example. ${ }^{1} \mathrm{H}$ NMR (500 MHz, Chloroform- $d$ ) $\delta$ : $7.44 \sim 7.31(\mathrm{~m}, 4 \mathrm{H}), 4.22(\mathrm{~s}, 2 \mathrm{H}), 3.13(\mathrm{tt}, J=11.7,3.7 \mathrm{~Hz}$, $1 \mathrm{H}), 2.88(\mathrm{~s}, 2 \mathrm{H}), 2.46(\mathrm{~s}, 3 \mathrm{H}), 2.20 \sim 2.04(\mathrm{~m}, 2 \mathrm{H}), 1.74$ (qd, $J=12.2,4.3 \mathrm{~Hz}, 2 \mathrm{H}), 1.48(\mathrm{~s}, 9 \mathrm{H}) ;{ }^{13} \mathrm{C}$ NMR $(126$ $\mathrm{MHz}$, Chloroform- $d$ ) $\delta$ : 172.26, 154.71, 147.35, 133.73, $130.72,130.37(2 \times \mathrm{C}), 129.64,128.90(2 \times \mathrm{C}), 79.65$, 43.71, $40.81(2 \times \mathrm{C}), 32.47(2 \times \mathrm{C}), 28.477(3 \times \mathrm{C}), 16.04$. HRMS (ESI) calcd for $\mathrm{C}_{20} \mathrm{H}_{25} \mathrm{ClN}_{2} \mathrm{O}_{2} \mathrm{SNa}[\mathrm{M}+\mathrm{Na}]^{+}$ 415.1395, found 415.1403 .

4.2.8 Preparation of 4-methyl-5-(substituted-phenyl)2-(piperidin-4-yl)thiazole hydrochlorates $(\mathbf{8 a} \sim \mathbf{8 p})$

In a $50 \mathrm{~mL}$ round bottom flask, tert-butyl 4-(4-methyl5-(substituted-phenyl)thiazol-2-yl)piperidine-1-carboxylate (7) $(0.05 \mathrm{~mol})$ were dissolved in $4 \mathrm{~mol} \cdot \mathrm{L}^{-1} \mathrm{HCl} /$ dioxane $(20 \mathrm{~mL})$, The mixture reacted at room temperature with stirring until no more solid produced. The mixture was filtered and washed with ether to give $\mathbf{8}$ directly used in the next reaction.

8g as an example. ${ }^{1} \mathrm{H}$ NMR $\left(500 \mathrm{MHz}, \mathrm{DMSO}-d_{6}\right) \delta$ : $9.38(\mathrm{~d}, J=9.0 \mathrm{~Hz}, 1 \mathrm{H}), 9.24(\mathrm{~d}, J=8.8 \mathrm{~Hz}, 1 \mathrm{H}), 7.56 \sim$ $7.46(\mathrm{~m}, 4 \mathrm{H}), 3.41 \sim 3.25(\mathrm{~m}, 3 \mathrm{H}), 3.07 \sim 2.95(\mathrm{~m}, 2 \mathrm{H})$, $2.40(\mathrm{~s}, 3 \mathrm{H}), 2.22 \sim 2.13(\mathrm{~m}, 2 \mathrm{H}), 2.05 \sim 1.90(\mathrm{~m}, 2 \mathrm{H}) ;{ }^{13} \mathrm{C}$ NMR $\left(126 \mathrm{MHz}, \mathrm{DMSO}-d_{6}\right) \delta$ : 168.23, 151.71, 134.32, $134.09,131.81,131.25,130.04,129.72,128.72,43.17$ $(2 \times \mathrm{C}), 37.00,29.00(2 \times \mathrm{C}), 17.35$. HRMS (ESI) calcd for $\mathrm{C}_{15} \mathrm{H}_{18} \mathrm{ClN}_{2} \mathrm{~S}[\mathrm{M}+\mathrm{H}]^{+}$293.0874, found 293.0882.

\subsubsection{Preparation of the target compounds $9 \mathbf{9} \sim 9 \mathbf{p}$}

In a $15 \mathrm{~mL}$ seal tube, 4-methyl-5-(substituted-phenyl)-2(piperidin-4-yl)thiazole hydrochlorates $(\mathbf{8 a} \sim \mathbf{8 p})(1 \mathrm{mmol})$, $N, N$-diisopropylethylamine $(5 \mathrm{mmol}), \mathrm{EDCl}(0.15 \mathrm{mmol})$, HOBT $(0.15 \mathrm{mmol})$ and 5-methyl-3-trifluoromethyl-1 $H$ pyrazol-1-acetic acid (3) (1.1 mmol) were dissolved in dichloromethane $(4 \mathrm{~mL})$. The mixture reacted at room temperature with stirring for $24 \mathrm{~h}$. The combined organic layers were washed with saturated $\mathrm{NaHCO}_{3}$ solution and brine, dried over with $\mathrm{MgSO}_{4}$ and concentrated under reduced pressure and column separation to give the target compounds $\mathbf{9 a} \sim \mathbf{9 p}$.

2-(5-Methyl-3-trifluoromethyl-1H-pyrazol-1-yl)-1-(4(4-methyl-5-phenylthiazol-2-yl)piperidin-1-yl)ethan-1-one (9a): White powder, yield 62\%. m.p. $113 \sim 114{ }^{\circ} \mathrm{C} ;{ }^{1} \mathrm{H}$ NMR (500 MHz, Chloroform- $d) \delta: 7.47 \sim 7.40(\mathrm{~m}, 4 \mathrm{H})$, $7.38 \sim 7.33(\mathrm{~m}, 1 \mathrm{H}), 6.35(\mathrm{~s}, 1 \mathrm{H}), 5.08 \sim 4.93(\mathrm{~m}, 2 \mathrm{H})$, $4.62(\mathrm{~d}, J=13.8 \mathrm{~Hz}, 1 \mathrm{H}), 4.06(\mathrm{~d}, J=14.2 \mathrm{~Hz}, 1 \mathrm{H}), 3.37 \sim$ $3.20(\mathrm{~m}, 2 \mathrm{H}), 2.98 \sim 2.83(\mathrm{~m}, 1 \mathrm{H}), 2.48(\mathrm{~s}, 3 \mathrm{H}), 2.33(\mathrm{~s}$, $3 \mathrm{H}), 2.22(\mathrm{dd}, J=24.2,12.2 \mathrm{~Hz}, 2 \mathrm{H}), 1.87 \sim 1.72(\mathrm{~m}, 2 \mathrm{H})$; ${ }^{13} \mathrm{C}$ NMR (126 MHz, Chloroform-d) $\delta: 170.45,163.76$, 147.14, 141.82, 141.74 (q, $J=37.8 \mathrm{~Hz}), 132.03,131.10$, $129.12(2 \times \mathrm{C}), 128.64(2 \times \mathrm{C}), 127.72,121.29(\mathrm{q}, J=$ $268.4 \mathrm{~Hz}), 104.31 \sim 104.04(\mathrm{~m}), 51.50,45.00,42.14$, 
40.21, 32.50, 31.92, 16.02, 11.22. HRMS (ESI) calcd for $\mathrm{C}_{22} \mathrm{H}_{24} \mathrm{~F}_{3} \mathrm{~N}_{4} \mathrm{OS}[\mathrm{M}+\mathrm{H}]^{+}$449.1617, found 449.1615.

2-(5-Methyl-3-trifluoromethyl-1H-pyrazol-1-yl)-1-(4(4-methyl-5-(4-(trifluoromethyl)phenyl)thiazol-2-yl)piperidin-1-yl)ethan-1-one (9b): White powder, yield 55\%. m.p. 148 $149{ }^{\circ} \mathrm{C} ;{ }^{1} \mathrm{H}$ NMR (500 MHz, Chloroform- $d$ ) $\delta: 7.69$ $(\mathrm{d}, J=8.2 \mathrm{~Hz}, 2 \mathrm{H}), 7.54(\mathrm{~d}, J=8.1 \mathrm{~Hz}, 2 \mathrm{H}), 6.35(\mathrm{~s}, 1 \mathrm{H})$, $5.09 \sim 4.95(\mathrm{~m}, 2 \mathrm{H}), 4.62(\mathrm{~d}, J=13.0 \mathrm{~Hz}, 1 \mathrm{H}), 4.07(\mathrm{~d}, J=$ $14.1 \mathrm{~Hz}, 1 \mathrm{H}), 3.36 \sim 3.21(\mathrm{~m}, 2 \mathrm{H}), 2.89(\mathrm{td}, J=14.3,13.0$, $3.0 \mathrm{~Hz}, 1 \mathrm{H}), 2.50(\mathrm{~s}, 3 \mathrm{H}), 2.34(\mathrm{~s}, 3 \mathrm{H}), 2.29 \sim 2.15(\mathrm{~m}$, 2H), $1.88 \sim 1.73(\mathrm{~m}, 2 \mathrm{H}) ;{ }^{13} \mathrm{C}$ NMR (126 MHz, Chloroform- $d$ ) $\delta: 171.45,163.80,148.31,141.84,141.74$ (q, $J=$ $38.0 \mathrm{~Hz}), 135.84,129.64(\mathrm{q}, J=32.7 \mathrm{~Hz}), 129.56,129.30$ $(2 \times \mathrm{C}), 125.62(\mathrm{q}, J=3.7 \mathrm{~Hz}, 2 \times \mathrm{C}), 123.95(\mathrm{q}, J=272.2$ $\mathrm{Hz}), 121.29$ (q, $J=268.6 \mathrm{~Hz}), 104.22 \sim 104.12(\mathrm{~m}), 51.47$, 44.97, 42.09, 40.24, 32.44, 31.88, 16.12, 11.19. HRMS (ESI) calcd for $\mathrm{C}_{23} \mathrm{H}_{23} \mathrm{~F}_{6} \mathrm{~N}_{4} \mathrm{OS}[\mathrm{M}+\mathrm{H}]^{+}$539.1311, found 539.1298.

1-(4-(5-(4-Fluorophenyl)-4-methylthiazol-2-yl)piperidin-1-yl)-2-(5-methyl-3-trifluoromethyl-1H-pyrazol-1yl)ethan-1-one (9c): White powder, yield 62\%. m.p. 133 $134{ }^{\circ} \mathrm{C} ;{ }^{1} \mathrm{H}$ NMR (500 MHz, Chloroform- $d$ ) $\delta: 7.46 \sim 7.33$ $(\mathrm{m}, 2 \mathrm{H}), 7.21 \sim 7.03(\mathrm{~m}, 2 \mathrm{H}), 6.35(\mathrm{~s}, 1 \mathrm{H}), 5.11 \sim 4.92(\mathrm{~m}$, 2H), $4.61(\mathrm{~d}, J=13.8 \mathrm{~Hz}, 1 \mathrm{H}), 4.10 \sim 3.99(\mathrm{~m}, 1 \mathrm{H}), 3.27$ (dddt, $J=26.6,11.3,7.7,3.5 \mathrm{~Hz}, 2 \mathrm{H}), 2.98 \sim 2.79(\mathrm{~m}, 1 \mathrm{H})$, $2.44(\mathrm{~s}, 3 \mathrm{H}), 2.33(\mathrm{~s}, 3 \mathrm{H}), 2.29 \sim 2.12(\mathrm{~m}, 2 \mathrm{H}), 1.88 \sim 1.69$ $(\mathrm{m}, 2 \mathrm{H}) ;{ }^{13} \mathrm{C}$ NMR (126 MHz, Chloroform-d) $\delta: 170.53$, 163.77, 162.28 (d, $J=248.1 \mathrm{~Hz}), 147.24,141.83,141.72$ $(\mathrm{d}, J=37.9 \mathrm{~Hz}), 130.86(\mathrm{~d}, J=8.2 \mathrm{~Hz}, 2 \times \mathrm{C}), 129.97$, $128.01(\mathrm{~d}, J=3.3 \mathrm{~Hz}), 121.28$ (q, $J=268.5 \mathrm{~Hz}), 115.67$ (d, $J=21.7 \mathrm{~Hz}, 2 \times \mathrm{C}), 104.23 \sim 104.10$ (m), 51.47, 44.98, 42.11, 40.19, 32.47, 31.90, 15.84, 11.19. HRMS (ESI) calcd for $\mathrm{C}_{22} \mathrm{H}_{22} \mathrm{~F}_{4} \mathrm{~N}_{4} \mathrm{OSNa}[\mathrm{M}+\mathrm{Na}]^{+}$489.1343, found 489.1334.

1-(4-(5-(4-Chlorophenyl)-4-methylthiazol-2-yl)piperidin-1-yl)-2-(5-methyl-3-trifluoromethyl-1H-pyrazol-1yl)ethan-1-one (9d): White powder, yield 66\%. m.p. 137 $138{ }^{\circ} \mathrm{C}$; ${ }^{1} \mathrm{H}$ NMR (500 MHz, Chloroform-d) $\delta: 7.41$ (d, $J=8.1 \mathrm{~Hz}, 2 \mathrm{H}), 7.35$ (d, $J=8.1 \mathrm{~Hz}, 2 \mathrm{H}), 6.35(\mathrm{~s}, 1 \mathrm{H})$, $5.11 \sim 4.92(\mathrm{~m}, 2 \mathrm{H}), 4.61(\mathrm{~d}, J=13.3 \mathrm{~Hz}, 1 \mathrm{H}), 4.13 \sim 4.01$ $(\mathrm{m}, 1 \mathrm{H}), 3.37 \sim 3.19(\mathrm{~m}, 2 \mathrm{H}), 2.94 \sim 2.82(\mathrm{~m}, 1 \mathrm{H}), 2.46(\mathrm{~s}$, $3 \mathrm{H}), 2.33(\mathrm{~s}, 3 \mathrm{H}), 2.29 \sim 2.13(\mathrm{~m}, 2 \mathrm{H}), 1.87 \sim 1.72(\mathrm{~m}$, $2 \mathrm{H}) ;{ }^{13} \mathrm{C}$ NMR (126 MHz, Chloroform- $\left.d\right) \delta: 170.95$, $163.81,147.45,141.85,141.77$ (d, $J=37.8 \mathrm{~Hz}), 133.84$, $130.45,130.35(2 \times \mathrm{C}), 129.93,128.90(2 \times \mathrm{C}), 121.29$ (q, $J=268.5 \mathrm{~Hz}), 104.30 \sim 104.08(\mathrm{~m}), 51.49,45.01,42.12$, 40.19, 32.49, 31.91, 15.94, 11.23. HRMS (ESI) calcd for $\mathrm{C}_{22} \mathrm{H}_{22} \mathrm{ClF}_{3} \mathrm{~N}_{4} \mathrm{OSNa}[\mathrm{M}+\mathrm{Na}]^{+}$505.1047, found 505.1033 .

1-(4-(5-(4-Bromophenyl)-4-methylthiazol-2-yl)piperidin-1-yl)-2-(5-methyl-3-trifluoromethyl-1H-pyrazol-1yl)ethan-1-one (9e): White powder, yield 72\%. m.p. 158 $159{ }^{\circ} \mathrm{C} ;{ }^{1} \mathrm{H}$ NMR (500 MHz, Chloroform- $d$ ) $\delta: 7.60 \sim 7.53$ $(\mathrm{m}, 2 \mathrm{H}), 7.32 \sim 7.26(\mathrm{~m}, 2 \mathrm{H}), 6.35(\mathrm{~s}, 1 \mathrm{H}), 5.08 \sim 4.94(\mathrm{~m}$, $2 \mathrm{H}), 4.64 \sim 4.57(\mathrm{~m}, 1 \mathrm{H}), 4.09 \sim 4.02(\mathrm{~m}, 1 \mathrm{H}), 3.35 \sim 3.20$ $(\mathrm{m}, 2 \mathrm{H}), 2.88(\mathrm{td}, J=14.2,13.2,3.1 \mathrm{~Hz}, 1 \mathrm{H}), 2.46(\mathrm{~s}, 3 \mathrm{H})$, $2.33(\mathrm{~s}, 3 \mathrm{H}), 2.26 \sim 2.15(\mathrm{~m}, 2 \mathrm{H}), 1.86 \sim 1.72(\mathrm{~m}, 2 \mathrm{H}) ;{ }^{13} \mathrm{C}$
NMR (126 MHz, Chloroform-d) $\delta: 170.95,163.81,147.52$, $141.85,141.80$ (q, $J=37.8 \mathrm{~Hz}), 131.87(2 \times \mathrm{C}), 130.97$, $130.63(2 \times \mathrm{C}), 129.94,121.96,121.29(\mathrm{q}, J=268.4 \mathrm{~Hz})$, 104.21 (q, $J=1.9 \mathrm{~Hz}$ ), 51.51, 45.03, 42.13, 40.21, 32.49, 31.91, 15.99, 11.25. HRMS (ESI) calcd for $\mathrm{C}_{22} \mathrm{H}_{22} \mathrm{BrF}_{3}$ $\mathrm{N}_{4} \mathrm{OSNa}[\mathrm{M}+\mathrm{Na}]^{+}$549.0542, found 549.0523.

1-(4-(5-(4-(tert-Butyl)phenyl)-4-methylthiazol-2-yl)piperidin-1-yl)-2-(5-methyl-3-trifluoromethyl-1H-pyrazol1-yl)ethan-1-one (9f): White powder, yield 50\%. m.p. 137 $138{ }^{\circ} \mathrm{C} ;{ }^{1} \mathrm{H}$ NMR (500 MHz, Chloroform- $d$ ) $\delta: 7.45$ $(\mathrm{d}, J=7.9 \mathrm{~Hz}, 2 \mathrm{H}), 7.36(\mathrm{~d}, J=8.0 \mathrm{~Hz}, 2 \mathrm{H}), 6.34(\mathrm{~s}, 1 \mathrm{H})$, $5.05 \sim 4.95(\mathrm{~m}, 2 \mathrm{H}), 4.61(\mathrm{~d}, J=14.2 \mathrm{~Hz}, 1 \mathrm{H}), 4.03(\mathrm{~d}, J=$ $14.6 \mathrm{~Hz}, 1 \mathrm{H}), 3.36 \sim 3.17(\mathrm{~m}, 2 \mathrm{H}), 2.94 \sim 2.82(\mathrm{~m}, 1 \mathrm{H})$, $2.48(\mathrm{~s}, 3 \mathrm{H}), 2.32(\mathrm{~s}, 3 \mathrm{H}), 2.26 \sim 2.15(\mathrm{~m}, 2 \mathrm{H}), 1.88 \sim 1.73$ $(\mathrm{m}, 2 \mathrm{H}), 1.36(\mathrm{~s}, 9 \mathrm{H}) ;{ }^{13} \mathrm{C}$ NMR $(126 \mathrm{MHz}$, Chloroform- $d)$ $\delta: 170.17,163.77,150.85,146.85,141.83,141.77$ (q, $J=$ $38.0 \mathrm{~Hz}), 131.16,129.06,128.79(2 \times \mathrm{C}), 125.61(2 \times \mathrm{C})$, 121.30 (q, $J=268.6 \mathrm{~Hz}), 104.26 \sim 104.13(\mathrm{~m}), 51.53$, $45.04,42.17,40.23,34.62,32.54,31.96,31.24(3 \times \mathrm{C})$, 16.06, 11.24. HRMS (ESI) calcd for $\mathrm{C}_{26} \mathrm{H}_{31} \mathrm{~F}_{3} \mathrm{~N}_{4} \mathrm{OSNa}$ $[\mathrm{M}+\mathrm{Na}]^{+}$527.2063, found 527.206.

1-(4-(5-(3-Chlorophenyl)-4-methylthiazol-2-yl)piperidin-1-yl)-2-(5-methyl-3-trifluoromethyl-1H-pyrazol-1yl)ethan-1-one (9g): White powder, yield 54\%. m.p. 126 $127{ }^{\circ} \mathrm{C} ;{ }^{1} \mathrm{H}$ NMR (500 MHz, Chloroform-d) $\delta: 7.46 \sim 7.29$ $(\mathrm{m}, 4 \mathrm{H}), 6.35(\mathrm{~s}, 1 \mathrm{H}), 5.08 \sim 4.95(\mathrm{~m}, 2 \mathrm{H}), 4.61(\mathrm{~d}, J=$ $14.3 \mathrm{~Hz}, 1 \mathrm{H}), 4.06(\mathrm{~d}, J=13.8 \mathrm{~Hz}, 1 \mathrm{H}), 3.37 \sim 3.20(\mathrm{~m}$, $1 \mathrm{H}), 2.89(\mathrm{td}, J=14.6,13.5,3.4 \mathrm{~Hz}, 1 \mathrm{H}), 2.47(\mathrm{~s}, 3 \mathrm{H})$, $2.33(\mathrm{~s}, 3 \mathrm{H}), 2.27 \sim 2.14(\mathrm{~m}, 2 \mathrm{H}), 1.87 \sim 1.72(\mathrm{~m}, 2 \mathrm{H}) .{ }^{13} \mathrm{C}$ NMR (126 MHz, Chloroform-d) $\delta: 171.03,163.79,147.89$, $141.83,141.75$ (d, $J=37.8 \mathrm{~Hz}), 134.51,133.84,129.89$, 129.57, 129.04, 127.81, 127.28, 121.28 (d, $J=268.4 \mathrm{~Hz})$, $104.38 \sim 103.99(\mathrm{~m}), 51.50,44.98,42.10,40.21,32.45$, $31.88,16.06,11.23$. HRMS (ESI) calcd for $\mathrm{C}_{22} \mathrm{H}_{22} \mathrm{ClF}_{3^{-}}$ $\mathrm{N}_{4} \mathrm{OSNa}[\mathrm{M}+\mathrm{Na}]^{+}$505.1047, found 505.1032.

2-(5-Methyl-3-trifluoromethyl-1H-pyrazol-1-yl)-1-(4(4-methyl-5-(m-tolyl)thiazol-2-yl)piperidin-1-yl)ethan-1one (9h): White powder, yield 42\%. m.p. 109 $110{ }^{\circ} \mathrm{C}$; ${ }^{1} \mathrm{H}$ NMR (500 MHz, Chloroform-d) $\delta: 7.37 \sim 7.12(\mathrm{~m}, 4 \mathrm{H})$, $6.35(\mathrm{~s}, 1 \mathrm{H}), 5.11 \sim 4.94(\mathrm{~m}, 2 \mathrm{H}), 4.67 \sim 4.55(\mathrm{~m}, 1 \mathrm{H})$, 4.05 (dd, $J=15.5,1.9 \mathrm{~Hz}, 1 \mathrm{H}), 3.35 \sim 3.21(\mathrm{~m}, 2 \mathrm{H}), 2.89$ $(\mathrm{td}, J=14.0,12.9,3.1 \mathrm{~Hz}, 1 \mathrm{H}), 2.48(\mathrm{~s}, 3 \mathrm{H}), 2.41(\mathrm{~s}, 3 \mathrm{H})$, $2.33(\mathrm{~s}, 3 \mathrm{H}), 2.28 \sim 2.13(\mathrm{~m}, 2 \mathrm{H}), 1.86 \sim 1.73(\mathrm{~m}, 2 \mathrm{H}) ;{ }^{13} \mathrm{C}$ NMR (126 MHz, Chloroform- $d$ ) $\delta: 170.35,163.77,146.98$, $141.82,141.74$ (d, $J=38.0 \mathrm{~Hz}), 138.37,131.89,131.24$, $129.83,128.53(2 \times \mathrm{C}), 126.19,122.36(\mathrm{t}, J=268.5 \mathrm{~Hz})$, $104.33 \sim 104.00(\mathrm{~m}), 51.49,45.00,42.14,40.18,32.51$, 31.92, 21.34, 16.02, 11.21. HRMS (ESI) calcd for $\mathrm{C}_{23} \mathrm{H}_{25} \mathrm{~F}_{3} \mathrm{~N}_{4} \mathrm{OSNa}[\mathrm{M}+\mathrm{Na}]^{+}$485.1593, found 485.1574.

2-(5-Methyl-3-trifluoromethyl-1H-pyrazol-1-yl)-1-(4(4-methyl-5-(3-(trifluoromethyl)phenyl)thiazol-2-yl)piperidin-1-yl)ethan-1-one (9i): White powder, yield 52\%. m.p. $130 \sim 131{ }^{\circ} \mathrm{C} ;{ }^{1} \mathrm{H}$ NMR (500 MHz, Chloroform-d) $\delta: 7.37$ (dddd, $J=9.4,7.7,5.0,2.2 \mathrm{~Hz}, 2 \mathrm{H}), 7.25 \sim 7.16(\mathrm{~m}, 2 \mathrm{H})$, $6.35(\mathrm{~s}, 1 \mathrm{H}), 5.11 \sim 4.95(\mathrm{~m}, 2 \mathrm{H}), 4.62(\mathrm{~d}, J=14.5 \mathrm{~Hz}$, $1 \mathrm{H}), 4.06(\mathrm{~d}, J=14.0 \mathrm{~Hz}, 1 \mathrm{H}), 3.36 \sim 3.23(\mathrm{~m}, 2 \mathrm{H}), 2.95 \sim$ $2.84(\mathrm{~m}, 1 \mathrm{H}), 2.38(\mathrm{~s}, 3 \mathrm{H}), 2.33(\mathrm{~s}, 3 \mathrm{H}), 2.29 \sim 2.17(\mathrm{~m}$, 
2H), $1.81(\mathrm{pd}, J=12.9,4.2 \mathrm{~Hz}, 2 \mathrm{H}) ;{ }^{13} \mathrm{C} \mathrm{NMR}(126 \mathrm{MHz}$, Chloroform- $d$ ) $\delta: 171.33,163.81,148.13,141.85,141.77$ (q, $J=38.0 \mathrm{~Hz}), 132.96,132.35,131.17$ (q, $J=32.6 \mathrm{~Hz})$, $129.50,129.24,125.80$ (q, $J=3.6 \mathrm{~Hz}), 124.44$ (q, $J=3.5$ $\mathrm{Hz}), 123.81(\mathrm{q}, J=272.4 \mathrm{~Hz}), 121.30(\mathrm{q}, J=268.5 \mathrm{~Hz})$, $104.58 \sim 103.82(\mathrm{~m}), 51.50,44.99,42.10,40.22,32.46$, $31.89,15.97,11.22$. HRMS (ESI) calcd for $\mathrm{C}_{23} \mathrm{H}_{22} \mathrm{~F}_{6} \mathrm{~N}_{4} \mathrm{OS}-$ $\mathrm{Na}[\mathrm{M}+\mathrm{Na}]^{+}$539.1311, found 539.1312.

1-(4-(5-(3-Bromophenyl)-4-methylthiazol-2-yl)piperidin-1-yl)-2-(5-methyl-3-trifluoromethyl-1H-pyrazol-1yl)ethan-1-one $(9 \mathbf{9 j})$ : White powder, yield $48 \%$. m.p. $140 \sim$ $141{ }^{\circ} \mathrm{C}$; ${ }^{1} \mathrm{H}$ NMR (500 MHz, Chloroform- $d$ ) $\delta: 7.56$ (s, $1 \mathrm{H}), 7.47(\mathrm{~d}, J=7.8 \mathrm{~Hz}, 1 \mathrm{H}), 7.34(\mathrm{~d}, J=7.8 \mathrm{~Hz}, 1 \mathrm{H})$, $7.32 \sim 7.26(\mathrm{~m}, 1 \mathrm{H}), 6.34(\mathrm{~s}, 1 \mathrm{H}), 5.05 \sim 4.93(\mathrm{~m}, 2 \mathrm{H})$, $4.60(\mathrm{~d}, J=14.2 \mathrm{~Hz}, 1 \mathrm{H}), 4.03(\mathrm{~d}, J=14.9 \mathrm{~Hz}, 1 \mathrm{H}), 3.36 \sim$ $3.17(\mathrm{~m}, 2 \mathrm{H}), 2.94 \sim 2.80(\mathrm{~m}, 1 \mathrm{H}), 2.46(\mathrm{~s}, 3 \mathrm{H}), 2.31(\mathrm{~s}$, $3 \mathrm{H}), 2.25 \sim 2.11(\mathrm{~m}, 2 \mathrm{H}), 1.86 \sim 1.70(\mathrm{~m}, 2 \mathrm{H}) ;{ }^{13} \mathrm{C} \mathrm{NMR}$ (126 MHz, Chloroform- $d$ ) $\delta:$ 171.06, 163.78, 147.92, $141.82,141.74$ (q, $J=37.8 \mathrm{~Hz}), 134.12,131.92,130.72$, $130.13,129.44,127.73,122.63,121.28$ (q, $J=268.5 \mathrm{~Hz}$ ), $104.32 \sim 104.02(\mathrm{~m}), 51.49,44.97,42.09,40.20,32.45$, 31.87, 16.04, 11.22. HRMS (ESI) calcd for $\mathrm{C}_{22} \mathrm{H}_{22} \mathrm{BrF}_{3}-$ $\mathrm{N}_{4} \mathrm{OSNa}[\mathrm{M}+\mathrm{Na}]^{+}$549.0542, found 549.0537.

1-(4-(5-(2,3-Dichlorophenyl)-4-methylthiazol-2-yl)piperidin-1-yl)-2-(5-methyl-3-trifluoromethyl-1H-pyrazol-1yl)ethan-1-one (9k): White powder, yield 67\%. m.p. 155 $156{ }^{\circ} \mathrm{C} ;{ }^{1} \mathrm{H}$ NMR $(500 \mathrm{MHz}$, Chloroform- $d$ ) $\delta: 7.60 \sim 7.48$ $(\mathrm{m}, 1 \mathrm{H}), 7.27(\mathrm{~d}, J=6.8 \mathrm{~Hz}, 2 \mathrm{H}), 6.35(\mathrm{~s}, 1 \mathrm{H}), 5.10 \sim 4.93$ $(\mathrm{m}, 2 \mathrm{H}), 4.62(\mathrm{~d}, J=12.6 \mathrm{~Hz}, 1 \mathrm{H}), 4.07(\mathrm{~d}, J=13.6 \mathrm{~Hz}$, $1 \mathrm{H}), 3.39 \sim 3.22(\mathrm{~m}, 2 \mathrm{H}), 2.96 \sim 2.82(\mathrm{~m}, 1 \mathrm{H}), 2.41 \sim 2.13$ $(\mathrm{m}, 8 \mathrm{H}), 1.80(\mathrm{td}, J=15.7,13.9,7.8 \mathrm{~Hz}, 2 \mathrm{H}) .{ }^{13} \mathrm{C} \mathrm{NMR}$ (126 MHz, Chloroform- $d$ ) $\delta$ : 172.11, 163.81, 150.03, $141.84,141.81$ (q, $J=38.4,38.0 \mathrm{~Hz}), 133.86,133.21$, $132.99,130.88,130.69,127.07,126.88,121.30$ (q, $J=$ $268.5 \mathrm{~Hz}), 104.31 \sim 104.14(\mathrm{~m}), 51.54,45.05,42.16$, $40.25,32.46,31.90,15.78,11.26$. HRMS (ESI) calcd for $\mathrm{C}_{22} \mathrm{H}_{21} \mathrm{Cl}_{2} \mathrm{~F}_{3} \mathrm{~N}_{4} \mathrm{OSNa}[\mathrm{M}+\mathrm{Na}]^{+}$539.0657, found 539.0655 .

1-(4-(5-(2,4-Dichlorophenyl)-4-methylthiazol-2-yl)piperidin-1-yl)-2-(5-methyl-3-trifluoromethyl-1H-pyrazol-1yl)ethan-1-one (9l): White powder, yield 66\%. m.p. 150 $151{ }^{\circ} \mathrm{C} ;{ }^{1} \mathrm{H}$ NMR (500 MHz, Chloroform-d) $\delta: 7.53$ (d, $J=1.9 \mathrm{~Hz}, 1 \mathrm{H}), 7.32 \sim 7.27(\mathrm{~m}, 2 \mathrm{H}), 6.35(\mathrm{~s}, 1 \mathrm{H}), 5.09 \sim$ $4.95(\mathrm{~m}, 2 \mathrm{H}), 4.62(\mathrm{~d}, J=14.3 \mathrm{~Hz}, 1 \mathrm{H}), 4.06(\mathrm{~d}, J=13.2$ $\mathrm{Hz}, 1 \mathrm{H}), 3.38 \sim 3.22(\mathrm{~m}, 2 \mathrm{H}), 2.98 \sim 2.80(\mathrm{~m}, 1 \mathrm{H}), 2.33(\mathrm{~s}$, $3 \mathrm{H}), 2.27(\mathrm{~s}, 5 \mathrm{H}), 1.90 \sim 1.71(\mathrm{~m}, 2 \mathrm{H}) ;{ }^{13} \mathrm{C}$ NMR $(126$ $\mathrm{MHz}$, Chloroform-d) $\delta: 172.19,163.78,150.10,141.82, \delta$ 141.77 (q, $J=38.0 \mathrm{~Hz}$ ), 135.40, 135.17, 133.36, 129.82, 129.32, 127.11, 125.98, 121.29 (q, $J=268.4 \mathrm{~Hz}$ ), 104.18 (q, $J=1.7 \mathrm{~Hz}), 51.50,45.01,42.12,40.23,32.43,31.87$, 15.76, 11.23. HRMS (ESI) calcd for $\mathrm{C}_{22} \mathrm{H}_{21} \mathrm{Cl}_{2} \mathrm{~F}_{3} \mathrm{~N}_{4} \mathrm{OSNa}$ $[\mathrm{M}+\mathrm{Na}]^{+}$539.0657, found 539.0651.

1-(4-(5-(2,5-Dichlorophenyl)-4-methylthiazol-2-yl)piperidin-1-yl)-2-(5-methyl-3-trifluoromethyl-1H-pyrazol1-yl)ethan-1-one (9m): White powder, yield 70\%. m.p. $146 \sim 147{ }^{\circ} \mathrm{C} ;{ }^{1} \mathrm{H}$ NMR (500 MHz, Chloroform- $\left.d\right) \delta$ : $7.47 \sim 7.29(\mathrm{~m}, 3 \mathrm{H}), 6.35(\mathrm{~s}, 1 \mathrm{H}), 5.09 \sim 4.93(\mathrm{~m}, 2 \mathrm{H})$,
4.60 (dd, $J=21.9,14.5 \mathrm{~Hz}, 1 \mathrm{H}), 4.06$ (d, $J=14.6 \mathrm{~Hz}, 1 \mathrm{H})$, $3.36 \sim 3.22(\mathrm{~m}, 2 \mathrm{H}), 2.94 \sim 2.84(\mathrm{~m}, 1 \mathrm{H}), 2.40 \sim 2.14(\mathrm{~m}$, $8 \mathrm{H}), 1.87 \sim 1.74(\mathrm{~m}, 2 \mathrm{H}) ;{ }^{13} \mathrm{C}$ NMR $(126 \mathrm{MHz}$, Chloroform- $d$ ) $\delta$ : $172.40,163.82,150.25,141.85, \delta 141.88(\mathrm{q}$, $J=37.9 \mathrm{~Hz}), 133.06,132.52,132.42,132.31,131.03$, $129.92,125.89,122.43$ (q, $J=278.3 \mathrm{~Hz}), 104.30 \sim 104.22$ (m), 51.59, 45.08, 42.17, 40.26, 32.49, 31.91, 15.83, 11.31 . HRMS (ESI) calcd for $\mathrm{C}_{22} \mathrm{H}_{21} \mathrm{Cl}_{2} \mathrm{~F}_{3} \mathrm{~N}_{4} \mathrm{OSNa}[\mathrm{M}+\mathrm{Na}]^{+}$ 539.0657 , found 539.0655 .

1-(4-(5-(2-Fluorophenyl)-4-methylthiazol-2-yl)piperidin-1-yl)-2-(5-methyl-3-trifluoromethyl-1H-pyrazol-1yl)ethan-1-one (9n): White powder, yield 32\%. m.p. 94 $95{ }^{\circ} \mathrm{C} ;{ }^{1} \mathrm{H}$ NMR (500 MHz, Chloroform-d) $\delta: 7.81 \sim 7.52$ $(\mathrm{m}, 4 \mathrm{H}), 6.35(\mathrm{~s}, 1 \mathrm{H}), 5.16 \sim 4.91(\mathrm{~m}, 2 \mathrm{H}), 4.62(\mathrm{~d}, J=$ $14.4 \mathrm{~Hz}, 1 \mathrm{H}), 4.07$ (d, $J=13.8 \mathrm{~Hz}, 1 \mathrm{H}), 3.44 \sim 3.19(\mathrm{~m}$, 2H), $2.97 \sim 2.80(\mathrm{~m}, 1 \mathrm{H}), 2.49(\mathrm{~s}, 3 \mathrm{H}), 2.34(\mathrm{~s}, 3 \mathrm{H}), 2.29 \sim$ $2.15(\mathrm{~m}, 2 \mathrm{H}), 1.90 \sim 1.73(\mathrm{~m}, 2 \mathrm{H}) ;{ }^{13} \mathrm{C} \mathrm{NMR}(126 \mathrm{MHz}$, Chloroform- $d$ ) $\delta$ : 172.06, 163.81, 159.69 (d, $J=248.9 \mathrm{~Hz})$, $149.63,141.85,141.83$ (q, $J=37.8 \mathrm{~Hz}), 132.09,132.07$, $130.17(\mathrm{~d}, J=8.1 \mathrm{~Hz}), 124.22(\mathrm{~d}, J=3.8 \mathrm{~Hz}), 121.30(\mathrm{~d}$, $J=268.5 \mathrm{~Hz}), 119.61(\mathrm{~d}, J=15.1 \mathrm{~Hz}), 116.12$ (d, $J=22.2$ $\mathrm{Hz}), 104.33 \sim 104.07(\mathrm{~m}), 51.56,45.06,42.18,40.23$, $32.51,31.91,15.97,11.28$. HRMS (ESI) calcd for $\mathrm{C}_{22} \mathrm{H}_{22}-$ $\mathrm{F}_{4} \mathrm{~N}_{4} \mathrm{OSNa}[\mathrm{M}+\mathrm{Na}]^{+}$489.1343, found 489.1344.

1-(4-(4-Cyclopropyl-5-(2-fluorophenyl)thiazol-2-yl)piperidin-1-yl)-2-(5-methyl-3-trifluoromethyl-1H-pyrazol1-yl)ethan-1-one (9o): White powder, yield 42\%. m.p. 109 $110{ }^{\circ} \mathrm{C} ;{ }^{1} \mathrm{H}$ NMR (500 MHz, Chloroform- $d$ ) $\delta: 7.50$ (td, $J=7.6,1.9 \mathrm{~Hz}, 1 \mathrm{H}), 7.36(\mathrm{tdd}, J=7.3,5.1,1.9 \mathrm{~Hz}$, $1 \mathrm{H}), 7.25 \sim 7.14(\mathrm{~m}, 2 \mathrm{H}), 6.34(\mathrm{~s}, 1 \mathrm{H}), 5.08 \sim 4.94(\mathrm{~m}$, $2 \mathrm{H}), 4.53$ (dt, $J=13.4,4.3 \mathrm{~Hz}, 1 \mathrm{H}), 4.00(\mathrm{dt}, J=13.6,4.6$ $\mathrm{Hz}, 1 \mathrm{H}), 3.34 \sim 3.16(\mathrm{~m}, 2 \mathrm{H}), 2.93$ (ddd, $J=14.0,11.6,3.1$ $\mathrm{Hz}, 1 \mathrm{H}), 2.32(\mathrm{~s}, 3 \mathrm{H}), 2.22 \sim 2.11(\mathrm{~m}, 2 \mathrm{H}), 1.94 \sim 1.84(\mathrm{~m}$, $1 \mathrm{H}), 1.84 \sim 1.72(\mathrm{~m}, 2 \mathrm{H}), 1.01(\mathrm{dt}, J=6.0,3.0 \mathrm{~Hz}, 2 \mathrm{H})$, $0.90(\mathrm{dt}, J=8.2,3.0 \mathrm{~Hz}, 2 \mathrm{H}) ;{ }^{13} \mathrm{C}$ NMR $(126 \mathrm{MHz}$, Chloroform- $d) \delta$ : 171.74, 163.77, $159.81(\mathrm{~d}, J=248.7 \mathrm{~Hz})$, $154.71,141.84,141.78(\mathrm{q}, J=37.8 \mathrm{~Hz}), 132.35(\mathrm{~d}, J=2.6$ $\mathrm{Hz}), 129.86$ (d, $J=8.1 \mathrm{~Hz}), 124.14(\mathrm{~d}, J=3.7 \mathrm{~Hz}), 121.31$ (q, $J=268.5 \mathrm{~Hz}) 119.79(\mathrm{~d}, J=15.2 \mathrm{~Hz}), 116.11,115.93$, $104.27 \sim 104.13(\mathrm{~m}), 51.57,44.92,42.04,39.99,32.32$, $31.78,11.25,10.99(\mathrm{~d}, J=2.0 \mathrm{~Hz}), 8.35(2 \times \mathrm{C})$. HRMS (ESI) calcd for $\mathrm{C}_{24} \mathrm{H}_{24} \mathrm{~F}_{4} \mathrm{~N}_{4} \mathrm{OSNa}[\mathrm{M}+\mathrm{Na}]^{+}$515.1499, found 515.1503 .

1-(4-(4-(2,6-Dichlorobenzyl)thiazol-2-yl)piperidin-1yl)-2-(5-methyl-3-trifluoromethyl-1 $H$-pyrazol-1-yl)ethan1-one (9p): White powder, yield 64\%. m.p. 142 $143{ }^{\circ} \mathrm{C}$; ${ }^{1} \mathrm{H}$ NMR (500 MHz, Chloroform- $d$ ) $\delta: 7.36(\mathrm{~d}, J=8.0 \mathrm{~Hz}$, $2 \mathrm{H}), 7.22 \sim 7.13(\mathrm{~m}, 1 \mathrm{H}), 6.51(\mathrm{~s}, 1 \mathrm{H}), 6.34(\mathrm{~s}, 1 \mathrm{H}), 5.05 \sim$ $4.94(\mathrm{~m}, 2 \mathrm{H}), 4.59 \sim 4.49(\mathrm{~m}, 1 \mathrm{H}), 4.46(\mathrm{~d}, J=1.1 \mathrm{~Hz}$, $2 \mathrm{H}), 4.02(\mathrm{~d}, J=14.5 \mathrm{~Hz}, 1 \mathrm{H}), 3.36 \sim 3.20(\mathrm{~m}, 2 \mathrm{H}), 2.95 \sim$ $2.81(\mathrm{~m}, 1 \mathrm{H}), 2.32(\mathrm{~s}, 3 \mathrm{H}), 2.26 \sim 2.11(\mathrm{~m}, 2 \mathrm{H}), 1.85 \sim$ $1.64(\mathrm{~m}, 2 \mathrm{H}) ;{ }^{13} \mathrm{C}$ NMR (126 MHz, Chloroform- $\left.d\right) \delta$ : $173.32,163.83,152.54,141.93,141.73$ (q, $J=37.9 \mathrm{~Hz})$. $136.02(2 \times \mathrm{C}), 134.89,128.55,128.33(2 \times \mathrm{C}), 121.30(\mathrm{~d}$, $J=268.5 \mathrm{~Hz}$ ), 113.00, 104.19 (q, $J=1.8 \mathrm{~Hz}), 51.45,44.90$, 42.04, 39.96, 33.35, 32.46, 31.86, 11.22. HRMS (ESI) calcd for $\mathrm{C}_{22} \mathrm{H}_{21} \mathrm{Cl}_{2} \mathrm{~F}_{3} \mathrm{~N}_{4} \mathrm{OSNa}[\mathrm{M}+\mathrm{Na}]^{+}$539.0657, found 


\subsection{2.}

\subsection{Biological activity test}

The bactericidal activity of the target compounds were tested by the in vitro plate method. Experimental strains were Fusarium graminearum, Diplocarpon mali, Rhizoctonia solani Ktihn, Phytophthora infestans and Botrytis cinerea. The experimental concentration was $100 \mu \mathrm{g} / \mathrm{mL}$ and azoxystrobin was used as a control drug. The quantitative agent of target compound was added at the designed concentration when the melted PDA medium was cooled to $60 \sim 70{ }^{\circ} \mathrm{C}$. After it was sufficiently cooled, the bacteria cake with a diameter of $5 \mathrm{~mm}$ was inoculated, and then the culture was carried out in an incubator. The colony diameter was measured and the growth inhibition rate was calculated after 4 days. Besides the results were investigated by the cross method.

Relative inhibition rate $(\%)=($ control sample diameter - treated group diameter)/control group expanded diameter $\times 100 \%$.

Supporting Information The ${ }^{1} \mathrm{H}$ NMR, ${ }^{13} \mathrm{C}$ NMR and HRMS for the synthesized compounds. The Supporting Information is available free of charge via the Internet at http://sioc-journal.cn.

\section{References}

[1] Hanagan, M. A.; Pasteris, R. J. WO 2009094407, 2009 [Chem. Abstr. 2009, 151, 234956].

[2] Cohen, Y.; Rubin, A. E.; Galperin, M. Phytoparasitica 2018, 46, 689.

[3] Miao, J. Q.; Chi, Y. D.; Lin, D.; Tyler, B. M.; Liu, X. L. Phytopathology. 2018, 108, 1412.

[4] Feng, X.; Wang, K.; Pan, L.; Xu, T.; Zhang, H.; Fantke, P. J. Agric. Food. Chem. 2018, 66, 8489.

[5] Kono, M.; Matsumoto, T.; Kawamura, T.; Nishimura, A.; Kiyota, Y.; Oki, H.; Miyazaki, J.; Lgaki, S.; Bdhnke, C. A.; Shimoji, M.; Koro, M. Bioorg. Med. Chem. 2013, 21, 28.

[6] Cohen, Y.; Rubin, A. E.; Galperin, M. Phytoparasitica 2018, 46, 689.

[7] Bittner, R. J.; Mila, A. L. Crop. Prot. 2017, 93, 9.

[8] Pasteris, R. J.; Hanagan, M. A.; Bisaha, J. J.; Finkelstein, B. L.; Hoffman, L. E.; Gregory, V.; Andreassi, J. L.; Sweigard, J. A.; Klyashchitsky, B. A.; Henry, Y. T.; Berger, R. A. Bioorg. Med. Chem. 2016, 24, 354.

[9] Yang, Z. H.; Tian, H.; Zhang, L. World Pestic. 2017, 39, 43 (in Chinese).
(杨子辉，田昊，张莉，世界农药, 2017, 39, 43.)

[10] StLaurent, D. R.; Romine, J. L. Synthesis 2009, 1445.

[11] Chen, L.; Zhu, Y. J.; Fan, Z. J.; Guo, Z. M.; Zhang, Z. M.; Xu, Z. H.; Song, Y. Q.; Yurievich, M. Y.; Belskaya, N. B.; Bakulev, V. A. J. Agric. Food Chem. 2017, 65, 745.

[12] Hu, D. J.; Liu, S. F.; Huang, T. H.; Tu, H. Y.; Zhang, A. D. Molecules 2009, 14, 1288

[13] Kamireddy, B.; Pasteris, R. J.; Hanagan, M. A. WO 2009094445, 2008 [Chem. Abstr. 2009, 151, 173451].

[14] Pasteris, R. J.; Hanagan, M. A. WO 2008013925, 2008 [Chem. Abstr. 2014, 160, 302215].

[15] Wu, Q. F.; Zhao, B.; Fan, Z. J.; Zhao, J. B.; Guo, X. F.; Yang, D. Y.; Zhang, N. L.; Yu, B.; Kalinina, T.; Glukhareva, T. RSC Adv. 2018, 8, 39593.

[16] Wu, Q. F.; Zhao, B.; Fan, Z. J.; Guo, X. F.; Yang, D. Y.; Zhang, N. L.; Yu, B.; Zhou, S.; Zhao, J. B.; Chen, F. J. Agric. Food Chem. 2019, 67, 1360.

[17] Pierre, C.; Nicola, R.; Tomoki, T.; Ulrike, W. N.; Arnd, V.; Juergen, B. WO 2010066353, 2010 [Chem. Abstr. 2010, 153, 62249].

[18] Pierre, P.; Nicola, R.; Stefan, H.; Tomoki, T.; Ulrike, T. W.; Arnd, V.; Pierre, W.; Sebastian, H.; Juergen, B. WO 2010037479, 2010 [Chem. Abstr. 2010, 152, 454087].

[19] Sulzermosse, S.; Cederbaum, F.; Lamberth, C.; Berthon, G.; Umarye, J.; Grasso, V.; Schlereth, A.; Blum, M.; Waldmeier, R. Bioorg. Med. Chem. 2015, 23, 2129.

[20] Choi, W. S.; Nam, S. W.; Kim, I. D.; Kim, S. H. J. Chem. 2015, 241793.

[21] Choi, W. S.; Nam, S. W.; Ahn, E. K. J. Korean Soc. Appl. Biol. Chem. 2010, 53, 206.

[22] Britta, O.; Stefan, H.; Pierre, W.; Martin, W.; Ulrike, W. N. WO 2015055574, 2015 [Chem. Abstr. 2015, 162, 560976].

[23] Hoemberger, G.; Ford, M. J. WO 2015181097, 2015 [Chem. Abstr. 2015, 164, 36949].

[24] Huang, G.; Yang, J. C.; Li, H. C.; Zhang, J.; Liu, C. L. Agrochemicals 2011, 50, 79 (in Chinese). (黄光, 杨吉春, 李慧超, 张静, 刘长令, 农药, 2011, 50,79.)

[25] Chen, S.; He, D. M.; Dong, X.; Cui, J. G.; Gan, C. F.; Huang, Y. M. Modern Agrochem. 2017, 16, 8 (in Chinese). (陈爽，何冬梅，董新，崔建国，甘春芳，黄燕敏，现代农药， 2017, 16, 8.)

[26] Li, L.; Chen, H.; Lin, Y. Synth. Commun. 2007, 37, 985.

[27] Hemming, K.; Khan, M.; Kondakal, V.; Pitard, A.; Amar, M.; Rice, C. ChemInform 2012, 43, 126.

[28] Zhu, F. Q; Aisa, H. A.; Zhang, J.; Sun, C. L.; He, Y.; Xie, Y. C.; Shen, J. S. Org. Proc. Res. Dev. 2018, 22, 91.

[29] Das, T.; Chakraborty, A.; Sarkar, A. Tetrahedron Lett. 2014, 55, 7198.

[30] Cristau, P.; Herrmann, S.; Rahn, N.; Voerste, A. WO 2009132785, 2009 [Chem. Abstr. 2009, 151, 491110].

[31] Dai, H.; Liu, J. B.; Tao, W. F.; Miao, W. K.; Fang, J. X.; Wang, Q. M. Chin. J. Org. Chem. 2016, 36, 393 (in Chinese). (戴红，刘建兵，陶伟峰，苗文科，方建新，汪清民，有机化学， 2016, 36. 393.) 